\title{
Hypercholesterolemia-induced increase in plasma oxidized LDL abrogated pro angiogenic response in kidney grafts
}

\author{
Thomas Kerforne ${ }^{1,2,3 \dagger}{ }^{2}$, Frédéric Favreau ${ }^{1,4,5 \dagger}$, Tackwa Khalifeh 1,6, Souleymane Maiga', Geraldine Allain 1,3,7, \\ Antoine Thierry ${ }^{1,3,8}$, Manuel Dierick ${ }^{9}$, Edouard Baulier ${ }^{1,3,10}$, Clara Steichen ${ }^{1,3 \ddagger}$ and Thierry Hauet ${ }^{1,3,10,11,12^{*} \neq}$
}

\begin{abstract}
Background: Renal transplantation is increasingly associated with the presence of comorbidity factors such as dyslipidemia which could influence the graft outcome. We hypothesized that hypercholesterolemia could affect vascular repair processes and promote post-transplant renal vascular remodeling through the over-expression of the antiangiogenic thrombospondin-1 interacting with vascular endothelial growth factor-A levels.

Methods: We tested this hypothesis in vitro, in vivo and in a human cohort using (1) endothelial cells; (2) kidney auto-transplanted pig subjected $(n=5)$ or not $(n=6)$ to a diet enriched in cholesterol and (3) a renal transplanted patient cohort (16 patients).

Results: Cells exposed to oxidized LDL showed reduced proliferation and an increased expression of thrombospondin-1. In pigs, 3 months after transplantation of kidney grafts, we observed a deregulation of the hypoxia inducible factor 1a-vascular endothelial growth factor-A axis induced in cholesterol-enriched diet animals concomitant with an overexpression of thrombospondin-1 and a decrease in cortical microvessel density promoting vascular remodeling. In patients, hypercholesterolemia was associated with decreased vascular endothelial growth factor-A plasma levels during early follow up after renal transplantation and increased chronic graft dysfunction.
\end{abstract}

Conclusions: These results support a potential mechanism through which a high fat-diet impedes vascular repair in kidney graft and suggest the value of controlling cholesterolemia in recipient even at the early stage of renal transplantation.

Keywords: Oxidized LDL, Kidney transplantation, Vascular remodeling

\section{Background}

In renal transplantation, the increase of older recipients due to population ageing amplifies the current organ shortage, promoting a raise in the mean donor age. These increases induce a higher prevalence of comorbidity factors which could influence renal graft outcome. During the transplantation process, kidney grafts are unavoidably exposed to ischemia-reperfusion (I/R) injury. The key

\footnotetext{
*Correspondence: thierry.hauet@gmail.com

${ }^{\dagger}$ Thomas Kerforne and Frédéric Favreau contributed equally to this work

${ }^{\ddagger}$ Clara Steichen and Thierry Hauet contributed equally to this work

${ }^{1}$ INSERM U1082 IRTOMIT, 2 rue de la Milétrie, CS90577, 86000 Poitiers,

France

Full list of author information is available at the end of the article
}

role played by $I / R$ in defining the balance between regenerative or detrimental pathways and irreversibly programming graft outcome imply that it is of paramount importance to determine the factors or co-morbidity factors which could early interact with graft viability.

Risk factors for cardiovascular diseases such as diabetes, hypertension and elevated plasmatic low-density lipoprotein (LDL) levels are well documented in renal diseases progression and also in transplantation or renal artery stenosis injury [1]. The high prevalence of dyslipidemia combined with the use of older donors and with the hyperlipidemic effects of immunosuppressors strengthens the link between hypercholesterolemia and kidney transplantation $[2,3]$. Thus, hyperlipidemia in the 
recipient is of greater importance related to calcineurin or MTOR inhibitor exposure given the subsequent exposure length of the graft to the recipient milieu. In general, hypercholesterolemia is associated with increased systemic levels of oxidized low density lipoproteins (OxLDL) well known to be involved in endothelial cell dysfunction $[4,5]$. Renal microcirculation, one of the critical targets of $\mathrm{I} / \mathrm{R}$ injury, plays a crucial role in the early regeneration phase but also in the extension of chronic renal disease through a complex interplay between proliferation, regeneration and capillary loss [6-8]. In healthy organs, vascular endothelial cells are protected from moderate injury by the release of autocrine signaling molecules such as vascular endothelial growth factor-A (VEGF-A) [9]. However, endothelial damage induced by $I / R$ affect this regenerative response $[10,11]$.

Previously, Cui et al. reported the role of thrombospondin-1 (TSP-1) as an important mediator for high fat-diet induced kidney dysfunction in mice characterized by macrophage infiltration and fibrosis development [12]. TSP-1 is also involved in numerous detrimental processes induced by kidney I/R, but little is known about this effect in kidney graft outcome and the link between TSP-1 and OxLDL [13]. TSP-1 is a matricellular glycoprotein that interacts with different receptors and has multiple functions including promotion of clot formation or fibrosis and is also well-known as an inhibitor of angiogenesis by different mechanisms such as interacting with VEGF-A levels or modulating the NO availability which may prevent kidney repair [14].

This study is aimed at determining the role of hypercholesterolemia and particularly oxidized LDL (OxLDL) in early vascular regenerative processes occurring after $\mathrm{I} / \mathrm{R}$ injury in renal transplantation that are distinct from the chronic injury of atherosclerosis induced by dyslipidemia. We hypothesized that OxLDL could impede protective angiogenesis mechanism induced by $I / R$ through the stimulation of TSP-1 expression and its effects on VEGF-A production.

\section{Methods}

\section{In vitro incubation of OxLDL on human aortic endothelial} cells

OxLDL's effects were evaluated in Human Aortic Endothelial Cells (HAEC) treated with culture medium supplemented or not with OxLDL $(25 \mu \mathrm{g} / \mathrm{mL})$ for $24 \mathrm{~h}$. Briefly, HAEC obtained from Gibco (Saint Aubin, France) were cultured in Medium 200 (M200, Gibco) supplemented with $10 \%$ fetal bovine serum (Invitrogen, Saint Aubin, France) in a humidified atmosphere at $5 \% \mathrm{CO}_{2}$ and $37^{\circ} \mathrm{C}$. Cells were serum starved for $12 \mathrm{~h}$ and then treated. After treatment, condition media was removed and cells were collected to western blotting analysis for protein expression of TSP-1 (1/500, Santa Cruz Biotechnology, Santa Cruz, California, USA), ADAMTS-1 (1/500, Santa Cruz Biotechnology, Santa Cruz, California, USA), PhosphoVEGF-R2 (1/1000, Cell signaling, Leiden, Netherlands), and VEGF-R2 (1/1000, Cell signaling, Leiden, Netherlands). TSP-1 levels in the supernatant were quantified by ELISA kit $(\mathrm{RnD}$ Systems, Minneapolis, USA). Flow cytometry analysis was used for alpha5beta3 integrin expression on HAEC cells (1/100, Millipore, Billerica, Massachusetts, USA). The effect of recombinant human TSP- $1(0.5 \mu \mathrm{g} / \mathrm{ml}$; RD System, Lille, France) on alpha5beta3 integrin protein expressions was investigated. Cell proliferation was assessed by the BrDU assay (Cell Proliferation ELISA, BrdU, Roche, USA) following the manufacturer's guidelines in cells treated with culture medium supplemented or not with OxLDL for $24 \mathrm{~h}$ in presence of recombinant VEGF (Gibco) and SiRNa targeted TSP-1 (Qiagen, Courtaboeuf, France).

\section{Animal model and surgical procedures}

Male Large White pigs (Sus Scrofa) were fed with a standard or a high-fat diet (standard diet $+20 \%$ lard and $2 \%$ cholesterol) immediately after weaning and maintained until euthanasia, i.e. 3 months after transplantation as previously described in previous studies $[1,15]$. Renal auto-transplantation model was performed 2 months after weaning as previously described in accordance with the institutional committee for the use and care of laboratory animals (CEEA Poitou-Charentes CE2012-4) [16]. Briefly, the left kidney was removed, flushed with $300 \mathrm{ml}$ of UW preservation solution and preserved at $4{ }^{\circ} \mathrm{C}$ in the same solution in static conditions for $24 \mathrm{~h}$. On the day of transplantation, the right kidney was removed and the left kidney grafted mimicking the nephron mass in transplanted situation. Two experimental groups were studied: Normal diet: transplanted kidneys collected 3 months after surgery from animals fed a standard diet $(n=6)$, Highfat diet: transplanted kidneys collected 3 months after surgery from animals fed a high-fat diet $(n=5)$. One transplanted high-fat diet pig died before completion of the study due to surgical complications and was not included in data analysis. Urinary and plasma creatinine or proteinuria were measured using an automatic analyzer (Modular, Roche Diagnostic, Meylan, France). Creatinine clearance was calculated by the formula: (Urinary volume $\times$ Urinary creatinine level)/plasma creatinine level. Peripheral blood was collected before kidney transplantation, at 3, 7 days and 1, 3 months after reperfusion. Urines were collected at 3, 7 days and 1,3 months after reperfusion. 


\section{Western blotting, immunohistochemistry and standard light microscopy}

These studies were performed on renal tissue from graft kidneys 3 months after transplantation to assess the mechanisms responsible for formation and maintenance of the renal microvasculature and vascular remodeling. We investigated by standard western blotting protocols [17] pro- angiogenic pathways with specific antibodies against hypoxia inducible factor 1a (HIF1a, 1/500, BD Biosciences, San Jose, California, USA), VEGF (1/500, Santa Cruz Biotechnology), Stromal cell-Derived Factor-1 (SDF-1, 1/1000, Abbiotech, San Diego, California, USA), factors involved in endothelial cells proliferation and migration: matrix metalloproteinase 9 (MMP-9, 1/1000, Millipore), alpha5beta3 integrin (1/1000, Millipore), and anti-angiogenic factor: A Disintegrin And Metalloproteinase with Thrombospondin Motif-1 (ADAMTS-1, 1/200, Santa Cruz Biotechnology). Loading controls were $\beta$ actin (1/20,000, Sigma, St Louis, Missouri, USA). Protein bands were revealed and intensities were quantified using AlphaEase FC software (Alpha Innotech Corporation, San Leandro, CA). Staining evaluations using semiquantitative analyses on cortex samples was performed as previously described and examined under blind conditions by a pathologist and a nephrologist [18]. Briefly, paraffin sections were used for tubulo-interstitial fibrosis evaluation by Masson trichrome staining. Alpha-smooth muscle actin ( $\alpha$ SMA, $1 / 100$, Dako, Stockholm, Sweden) expression was assessed by diamino benzidine staining. The microvascular media-to-lumen ratio was measured in $\alpha$ SMA-positive macrovessels under $500 \mu \mathrm{m}$ in diameter. Tubular atrophy was assessed by HematoxylinEosin-Safran (HES) staining with previously published scoring [19]. Frozen sections were used for TSP-1 (1/100, Santa Cruz Biotechnology), VEGF (1/100, Santa Cruz Biotechnology), HIF1a (1/100, BD Biosciences) expressions in cortical section by immunofluorescence as well as ED-1 antigen marker of macrophages and monocytes (1/100, AbD Serotec, Oxford, UK).

\section{Apoptotic signals}

Apoptotic renal cells were characterized by the TUNEL method using the DeadEnd Fluorometric TUNEL system (Promega, Fitchburg, Massachusetts, USA) as previously shown [20].

\section{Real-time quantitative PCR}

We used RNA extraction kit (Qiagen, Courtaboeuf, France). Genomic DNA was removed using DNA-free kit (Applied Biosystems, Foster City, California, USA) and first-strand reverse transcription (Applied) was performed. Real-Time PCR assays were performed on an
ABI Prism 7300 (Applied) with porcine primers adapted for VEGF and TSP-1 mRNA expressions (Additional file 1: Table S1).

\section{Tissue preparation, acquisition and analysis of images by high resolution micro-computed tomography} Three months post-transplant at the time of sacrifice, the kidney graft was removed and perfused with a saline solution containing $5000 \mathrm{IU} / \mathrm{L}$ of heparin. Then, the saline solution was replaced by an intravascular radioopaque silicone polymer (Microfil MV122; Flow Tech, Carver, Massachusetts, USA). The kidney was immersed in formalin solution at $4{ }^{\circ} \mathrm{C}$ and a cylinder biopsy (diameter: $1 \mathrm{~cm}$; depth: $1.5 \mathrm{~cm}$ ) of the polymer-filled kidney was performed and encased in paraffin. High resolution micro-computed tomography scans were performed, followed by image reconstructions using specific algorithm [21]. The average diameter and spatial density of cortical vascular segments of microvessels were calculated as previously described $[17,22]$ and the spatial density classified according to diameter as small $(<40 \mu \mathrm{m})$, medium $(40-80 \mu \mathrm{m})$, large $(80-120 \mu \mathrm{m})$ or very large $(>120 \mu \mathrm{m})$ vessels.

\section{Patients}

A prospective cohort study was conducted on 16 kidney transplant patients at the Transplant Unit of the Poitiers University Hospital between January 2010 and June 2010. Adult recipients of a first or second kidney transplant were eligible for enrollment and patients with preemptive graft, infectious complications and early acute graft rejection have been excluded. The blood samples were taken during the normal follow-up of the patient, anonymized and since the study did not require additional blood sampling, an approval from an ethics committee was not required under French law according to the article L.1121-1 of the public health code. Written informed consents were obtained from each patient according to the Declaration of Helsinki. We classified patients in 2 groups related to the plasma cholesterol levels using a threshold of $1.80 \mathrm{~g} / \mathrm{L}$. Renal function was assessed by urinary protein excretion and eGFR according to the simplified modification of diet in renal disease formula MDRD. Peripheral blood was collected by venipuncture at day 0 (D0) before kidney transplantation, 1 , 3, 7 and 14 days (respectively D1, D3, D7 and D14), 1, 3 and 12 months after surgery. Plasma samples were stored at $-80{ }^{\circ} \mathrm{C}$ prior to protein quantification by ELISA for OxLDL (Mercodia, France), and VEGF-A (RD System).

\section{Statistics}

Results are shown as means \pm SD. We used a student t-test for two-group comparisons or a Mann-Whitney 
test when the variance was not equal between the groups. For multiple group comparison, we used Kruskal-Wallis test. Fisher's exact test was used for statistical analysis of proportions. Statistical significance was accepted for $\mathrm{p}<0.05$.

\section{Results}

The inhibition of endothelial cells proliferation induced by OxLDL is mediated by TSP-1

OxLDL-enriched culture medium induced the expression of ADAMTS-1 in HAEC and promoted the increase of TSP-1 secretion in supernatant without effect on phosphorylation of VEGF-R2 (Fig. 1a, c). Alpha5beta3 integrin expression on HAEC, marker of endothelial cell proliferation, was decreased in both conditions: in culture medium enriched with OxLDL or supplemented with TSP-1 human recombinant protein (Fig. 1b). Interestingly, SiRNA targeted TSP-1 (Additional file 2: Figure S1) limited the inhibition of cell proliferation induced by OxLDL in presence of VEGF underlining the role of TSP-1 in these inhibited conditions (Fig. 1d).

\section{High-fat diet reduces pro-angiogenic pathways in renal auto-transplanted pig}

In a clinical relevant porcine model, as previously described, hyperlipidic diet induced an increase of plasma cholesterol level in the last part of the follow up even if OxLDL plasmatic levels were significantly elevated at the day of surgical procedure and at 3 months post-transplantation [15]. As previously reported, only high-fat diet was associated with an increased proteinuria at 1 and 3 months while both experimental conditions, with or without high-fat diet, induced similar kinetics for creatinine clearance (Table 1) [15]. The effects of OxLDL on regenerative vascular processes suggested by our in vitro results were further assessed by measuring the expressions of proteins involved in these pathways at 3 months postreperfusion. The hypoxic pathway mediated by HIF1a

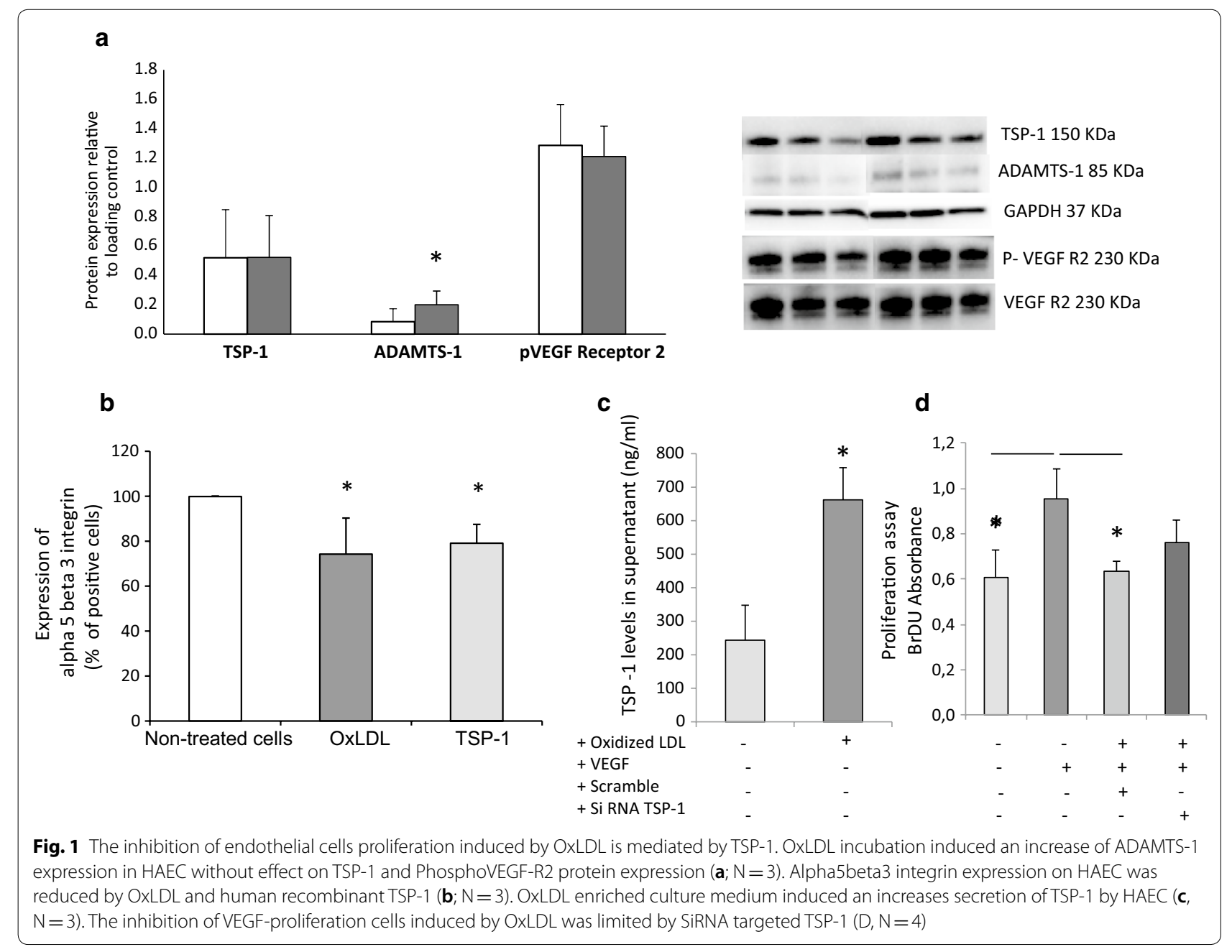


Table 1 Summary table of kidney function and blood metabolites of transplanted animals fed either a normal or a high-fat diet maintained for $\mathbf{3}$ months after surgery (M3)

\begin{tabular}{|c|c|c|c|c|c|}
\hline & Do & D3 & D7 & M1 & M3 \\
\hline \multicolumn{6}{|c|}{ Creatinine clearance (ml/min) } \\
\hline Normal diet & / & $7.8 \pm 7.5$ & $22.1 \pm 3.3$ & $71.9 \pm 22.3$ & $64.5 \pm 35.3$ \\
\hline High-fat diet & / & $8.6 \pm 14.3$ & $24.7 \pm 26.7$ & $61.4 \pm 22.3$ & $58.5 \pm 26.1$ \\
\hline \multicolumn{6}{|c|}{ Urinary ratio protein/creatinine (mg/mmol) } \\
\hline Normal diet & / & / & l & $124 \pm 5$ & $108 \pm 4$ \\
\hline High-fat diet & / & / & / & $236 \pm 12^{*}$ & $183 \pm 9^{*}$ \\
\hline
\end{tabular}

Values are mean \pm SD

* $p<0.05$ vs. normal diet, $n=5-6$ in each group

well known to promote VEGF production was abrogated by the high-fat diet. Dyslipidemic pigs exhibited an increased expression of HIF1a in kidney grafts 3 months after while VEGF-A expression remained stable or even decreased when assessed by western blotting and immunohistochemistry respectively (Figs. 2, 3, 4a, b, Table 2, Additional file 3: Figure S2, Additional file 4: Figure S3). In addition, high-fat diet increased the expression of ADAMTS-1 (Fig. 4a) known as an activator of the TSP-1 pathway and decreased the expression of proteins involved in endothelial cell proliferation and migration such as SDF-1 (Fig. 4b). A trend was observed for MMP-9 and alpha5beta3 integrin (Fig. 4b).

TSP-1 pathway is activated by high-fat diet in kidney graft To investigate the role of TSP-1 in the inhibition of the HIF1a pro-angiogenic pathways induced by hyperlipidic diet, we assessed TSP-1 expression in kidney graft. We observed in the high-fat diet group an increased protein expression of TSP-1 (Fig. 5; Table 2, Additional file 5: Figure S4). These results observed by immunohistological studies showed an elevated staining of TSP-1 in the interstitium, tubules and glomerulus in high-fat diet group but not significant difference in glomerulus parietal cell (Table 2). High-fat diet did not affect VEGF-A and TSP-1 mRNA expressions indicating rather a posttranscriptional regulation of these pathways (Additional file 6: Figure S5).

\section{High-fat diet induces vascular and tissue remodeling in kidney graft}

The increase of macrovascular media-to-lumen ratio indicated an intensive vascular remodeling in high-fat diet group compared to normal group (Fig. 6A). In addition, tubular atrophy was increased suggesting deleterious mechanism induced by hyperlipidic diet resulting in non functional fibrous tissue development assessed by Masson trichrome staining (Fig. 6B, C; Table 2) as
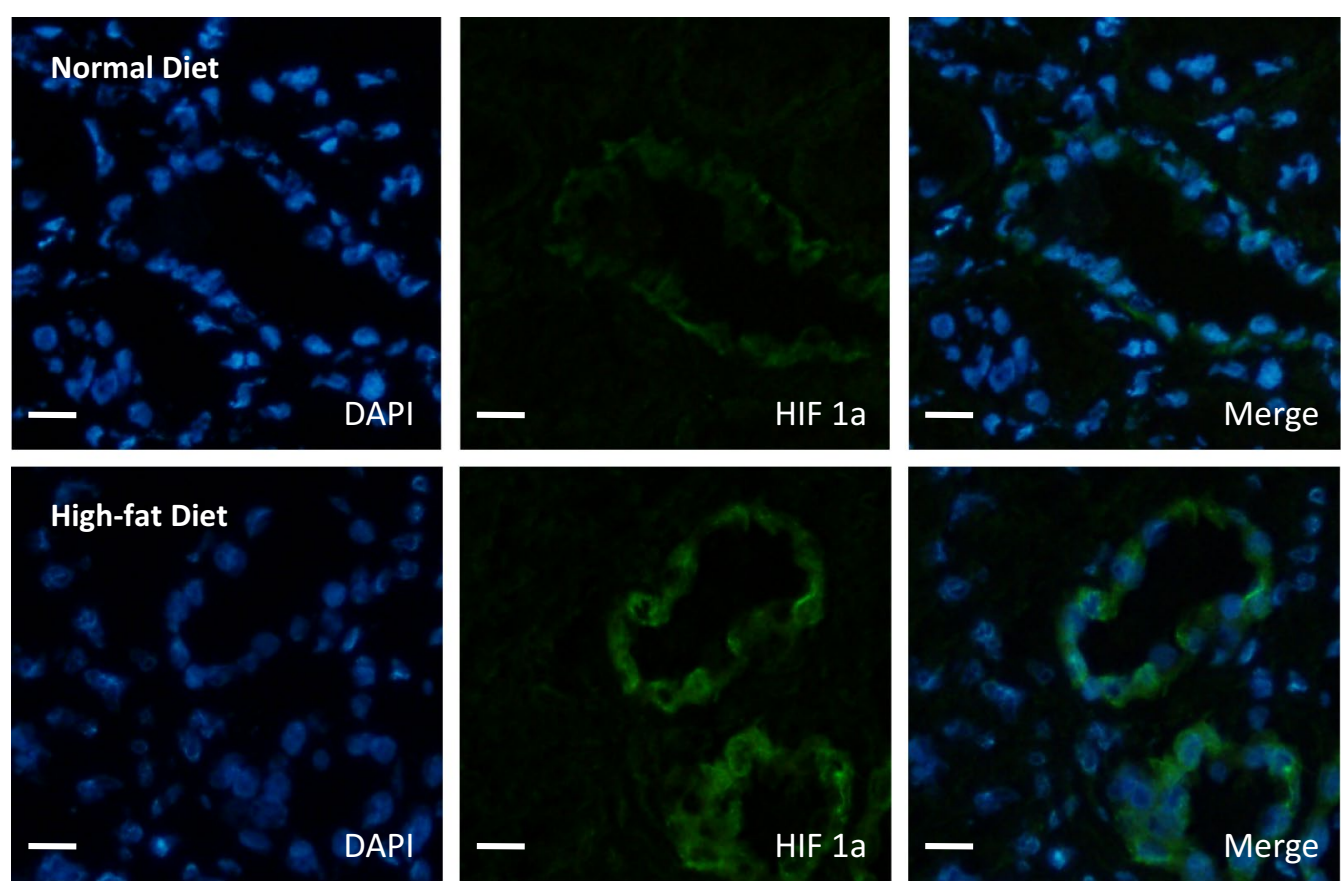

Fig. 2 High-fat diet increases HIF1a expression in porcine auto-transplanted kidneys. Immunofluorescence staining in cortex tissue for HIF1a in high-fat or normal diet groups, 3 months after renal auto-transplantation surgery. Scale bars represent $100 \mu \mathrm{m}$ (magnification $\times 40 ; n=5$ in each group) 

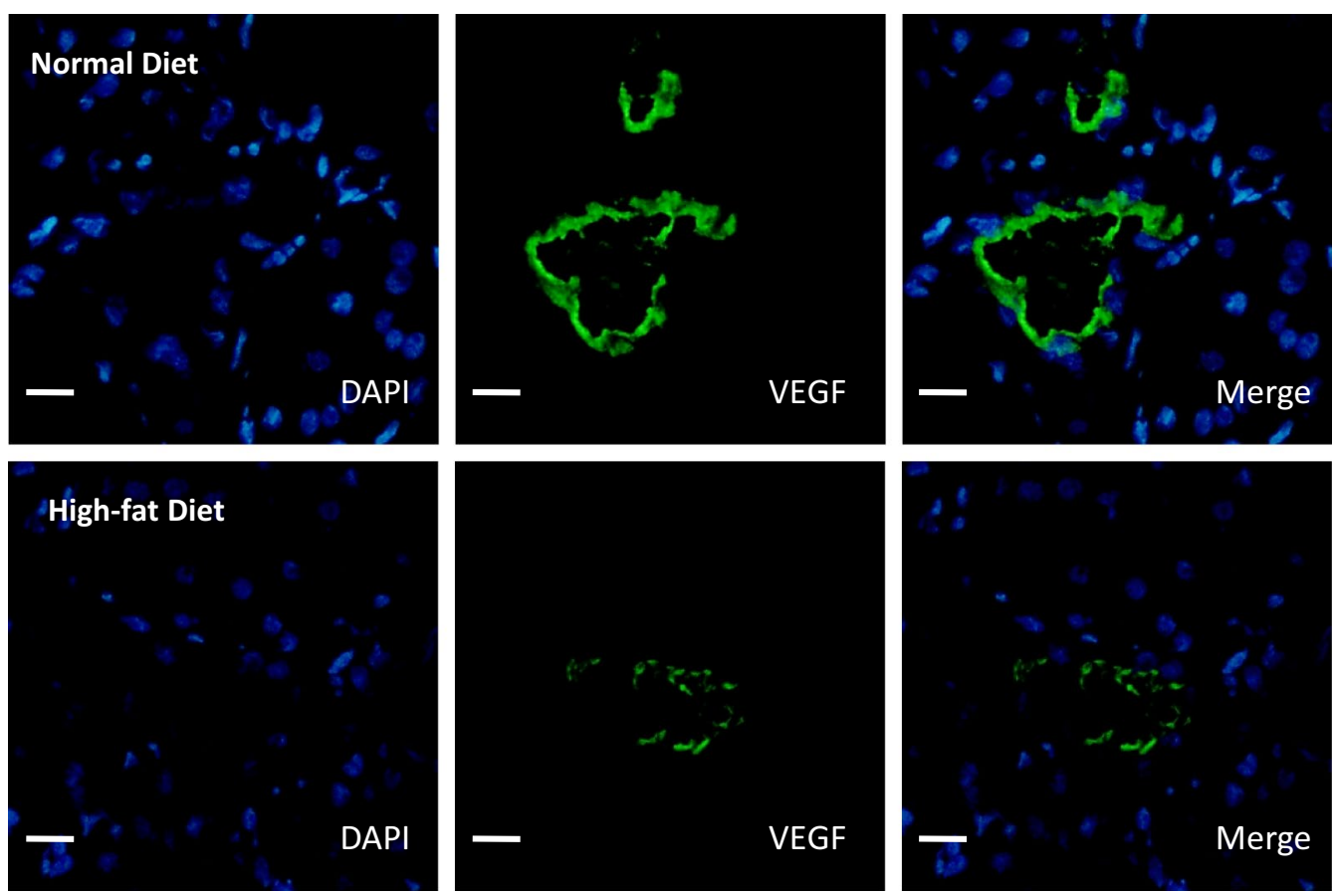

Fig. 3 High-fat diet reduces VEGF expression in porcine auto-transplanted kidneys. Immunofluorescence staining in cortex tissue for VEGF in high-fat or normal diet groups, 3 months after renal auto-transplantation surgery. Scale bars represent $100 \mu$ m (magnification $\times 40 ; n=5$ in each group)
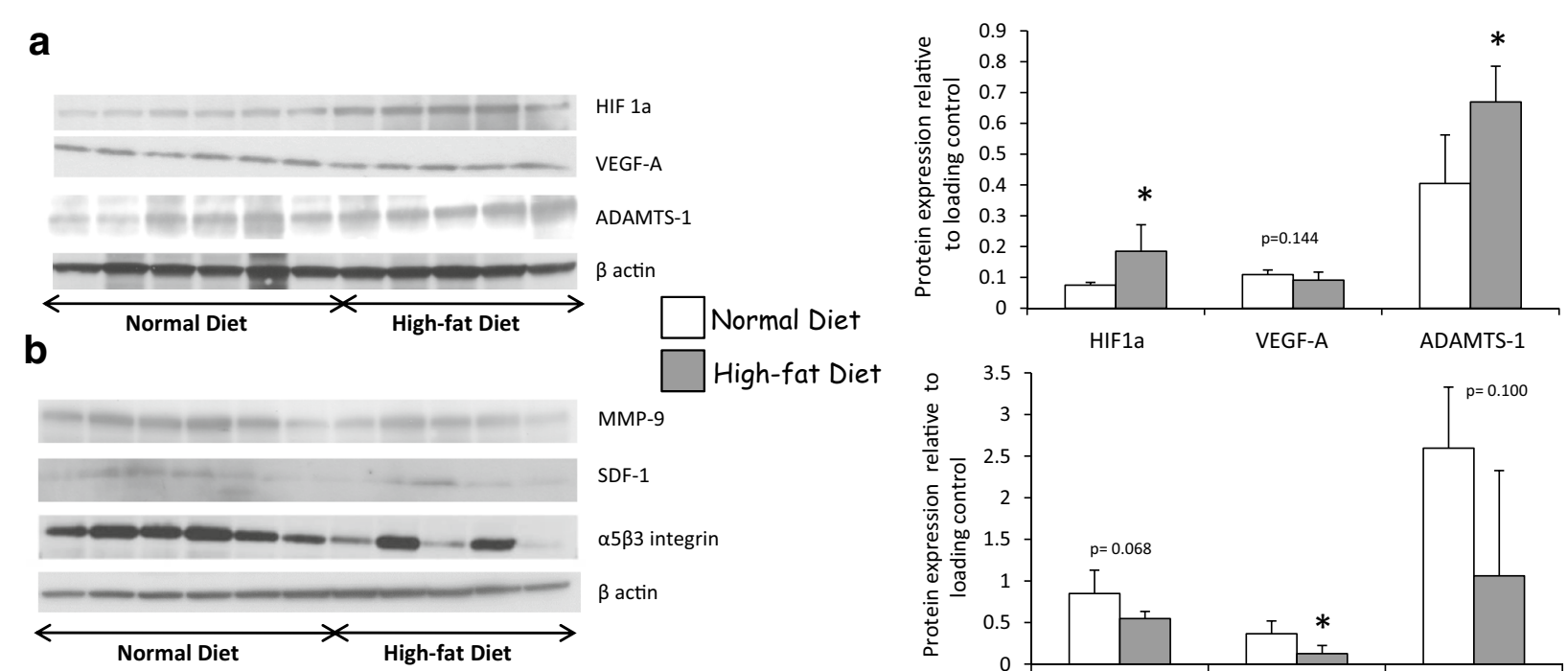

High-fat Diet

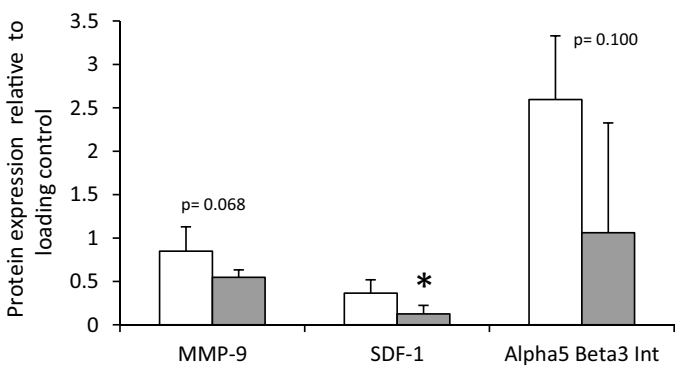

Fig. 4 High-fat diet reduces pro-angiogenic pathways in porcine auto-transplanted kidneys. Expression by western blotting of proteins involved in pro-angiogenic pathways: HIF1a, VEGF-A, ADAMTS-1, MMP-9, SDF-1, alpha5beta3 integrin (a, b). Values significantly different from the normal diet group are represented by ${ }^{*} p<0.05 ; n=5$ in high-fat diet and $n=6$ in normal diet group 
Table 2 Summary table of semi-quantification of kidney stainings in transplanted animals fed either a normal or a high-fat diet maintained for $\mathbf{3}$ months after surgery (M3)

\begin{tabular}{lcc}
\hline & Normal diet & High-fat diet \\
\hline $\begin{array}{l}\text { Renal HIF1a expression by field } \\
\quad\end{array}$ & $5.40 \pm 0.53$ & $11.52 \pm 0.58^{*}$ \\
$\quad$ Positive tubules & $5.00 \pm 0.63$ & $11.88 \pm 1.00^{*}$ \\
Positive cells & $6.00 \pm 0.35$ & $13.70 \pm 0.91^{*}$ \\
$\quad$ Surface area (\%) & $6.98 \pm 0.47$ & $2.78 \pm 0.32^{*}$ \\
Renal VEGF-A expression by field & & \\
$\quad$ positive vessels & & \\
Renal TSP-1 expression by field & & \\
$\quad$ Positive glomerulus parietal cell & $2.54 \pm 0.28$ & $2.72 \pm 0.35$ \\
Positive glomerulus & $1.73 \pm 0.29$ & $3.12 \pm 0.28^{*}$ \\
$\quad$ Positive tubules & $2.69 \pm 0.47$ & $9.03 \pm 1.39^{*}$ \\
Interstitium staining (\%) & $4.00 \pm 0.63$ & $16.83 \pm 0.70^{*}$ \\
Tubular atrophy (\%) & $6.35 \pm 1.30$ & $16.80 \pm 0.74^{*}$ \\
Trichrome staining (\%) & $6.31 \pm 0.78$ & $16.81 \pm 0.47^{*}$ \\
\hline
\end{tabular}

Values are mean $\pm S D$

${ }^{*} \mathrm{p}<0.05$ vs. normal diet, $\mathrm{n}=5$ in each group

previously suggested [15]. High resolution micro-computed tomography showed a decrease of the density of vascular segments with a diameter inferior to $40 \mu \mathrm{m}$ in the hyperlipidic diet kidney grafts, indicating a high microvessel injury (Fig. 6D). These results were supported by aminopeptidase staining indicating a decrease of capillary density in hyperlipidic diet group (Additional file 7; Additional file 8: Figure S6). In the cortex, this experimental group exhibited a reduction of the average vascular segment diameter supporting a vascular remodeling stimulated by high-fat diet (Fig. 6D). These injuries were associated with a pro-inflammatory milieu supported by ED1-positive cells infiltration significantly elevated in dyslipidemic pigs as well as apoptosis stimulation (Fig. 7A, B).

\section{Hypercholesterolemia the day of surgery affects VEGF-A secretion and promotes chronic graft dysfunction in patients}

This single-center study enrolled a total of 16 Caucasian renal transplant recipients. Characteristics of recipients and renal transplant allograft donors are listed in Table 3. All patients received a kidney graft from deceased donors. Using a threshold of $1.80 \mathrm{~g} / \mathrm{L}$ of plasma cholesterol level, the day before renal transplantation, we classified patients in hyper or normocholesterolemic groups (Fig. 8a). There was no patient death and two grafts loss during the follow up (1 in each group). Plasma OxLDL measurement in the blood samples collected before surgery showed a high level in
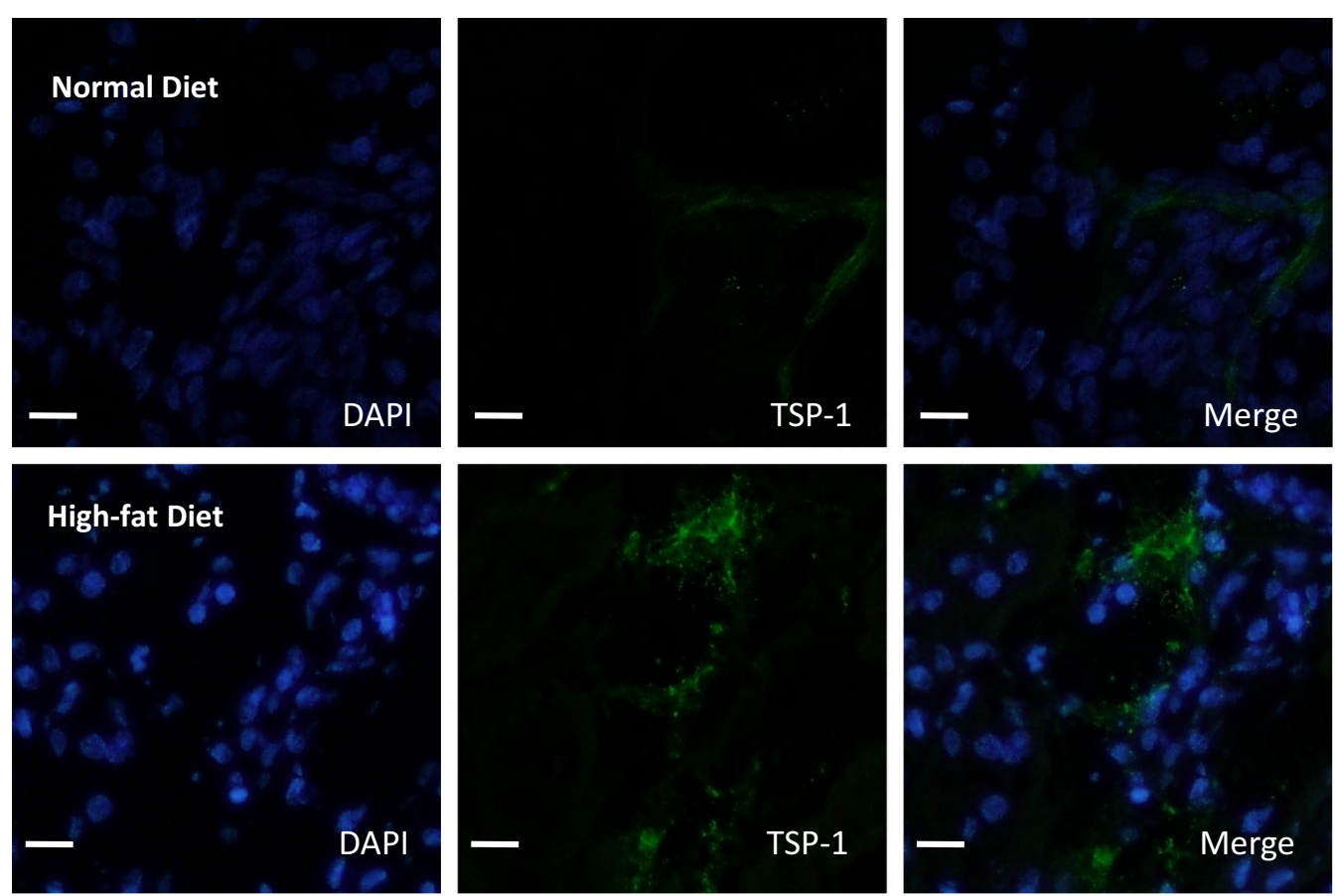

Fig. 5 High-fat diet promotes TSP-1 expression in porcine auto-transplanted kidney. TSP-1 expression by immunofluorescence staining was assessed in cortical part from the kidney grafts subjected or not to a hyperlipidic diet. Scale bars represent $100 \mu m($ Magnification $\times 40 ; n=5$ in each group) 


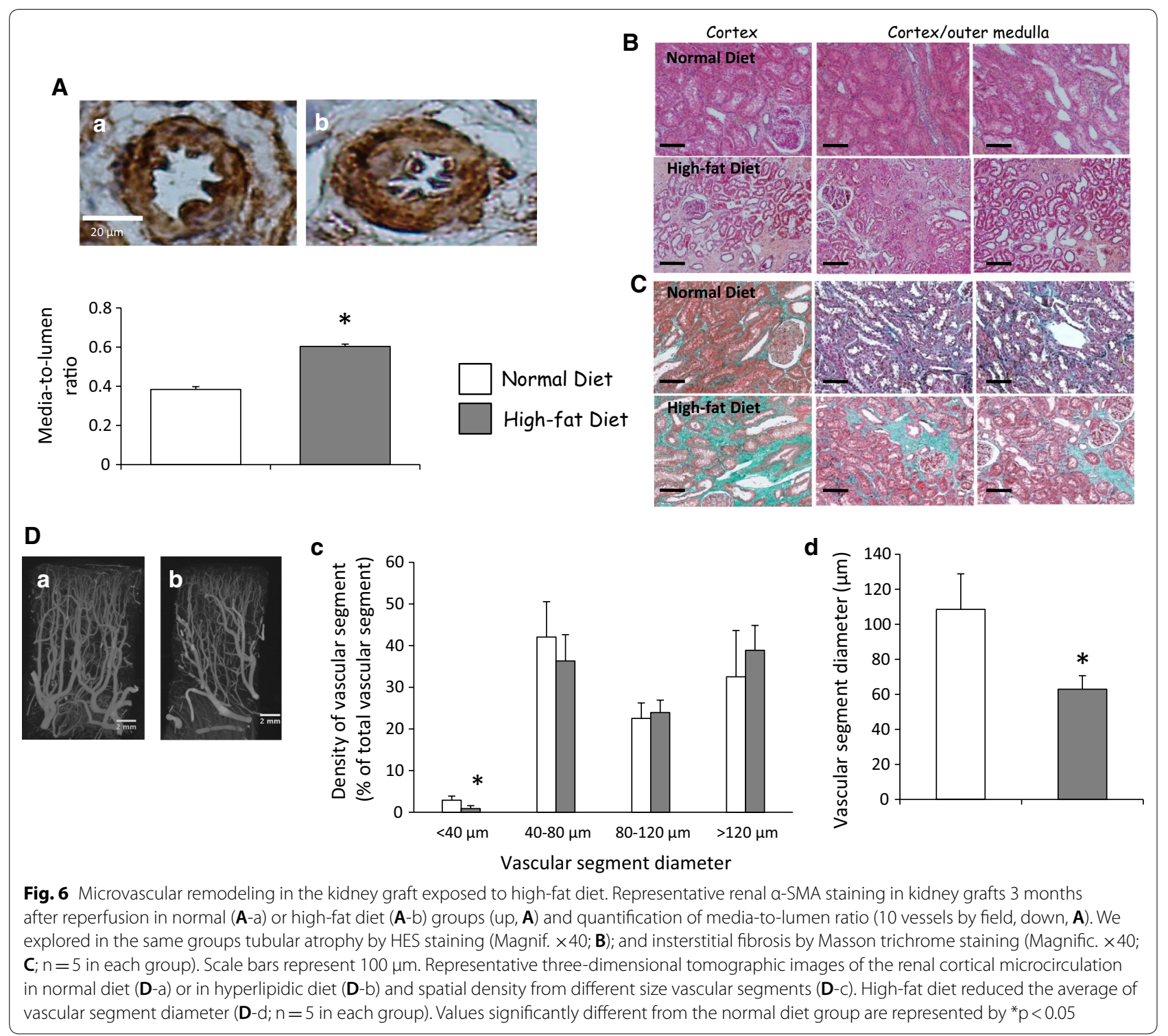

the hypercholesterolemia group but not reached statistical difference $(\mathrm{p}=0.061$; Fig. $8 \mathrm{~b})$. Interestingly, we observed a decrease of plasmatic VEGF-A level, 1 day after surgery in the hypercholesterolemia group which reached statistical significance at D3 and returned to basal level from D7 (Fig. 8c). The levels of plasma TSP-1 before surgery were increased in the hypercholesterolemia group (Additional file 9: Figure S7). Estimated glomerular filtration rate (eGFR) was similar in both groups up to 3 months post-surgery. However, 1 year after transplantation, the eGFR was significantly reduced in the hypercholesterolemia group (Table 3 ) and urinary protein excretion increased (Table 3).

\section{Discussion}

Organ shortage in renal transplantation has two major consequences: (1) it pushes transplantation centers to use organs obtained from older donors; (2) it increases the time spent on the waiting list, therefore increasing the average recipient age. This type of donors/recipients is linked with age-related co-morbidity factors such as hypertension or hypercholesterolemia, negatively affecting the transplantation outcome and leading back patients to dialysis [23]. The identification of involved mechanisms could improve the management of recipient in these conditions and promote new therapeutic strategies. Previously, Cui et al. reported for the first time that 
A

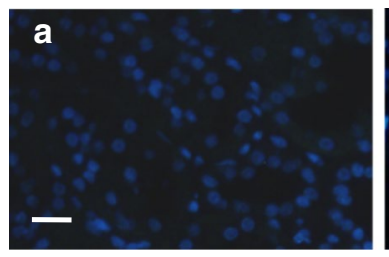

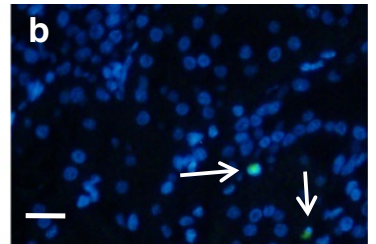

B

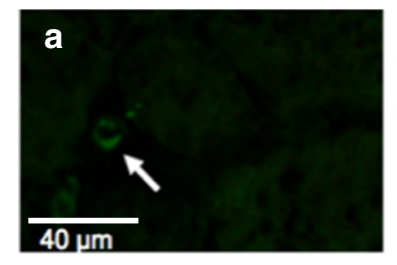

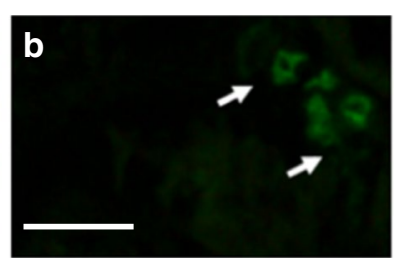
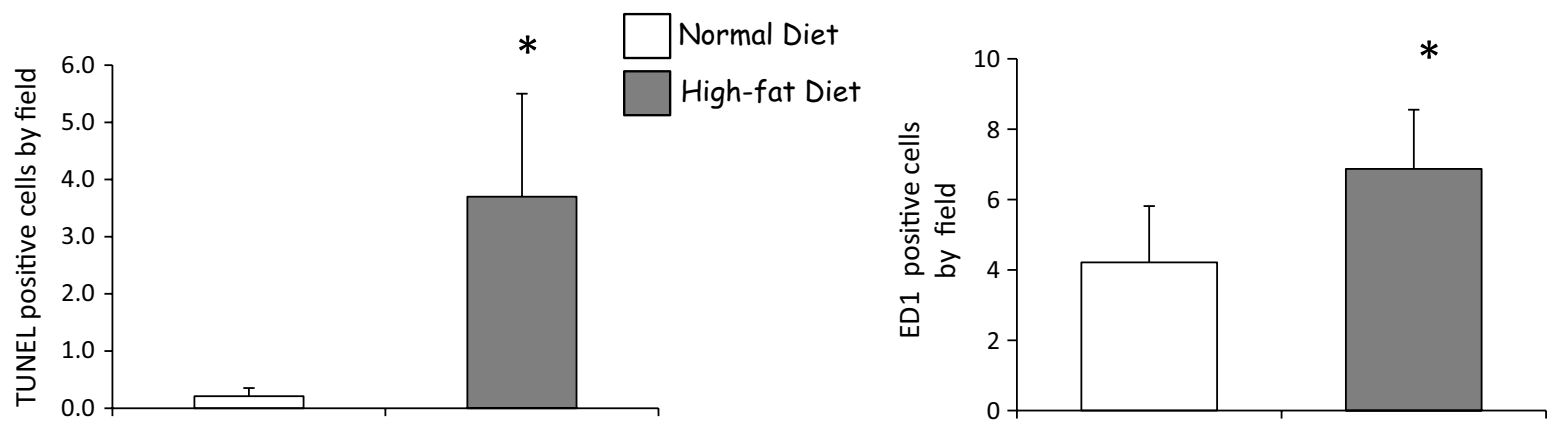

Fig. 7 High-fat diet promotes apoptosis and macrophage infiltration in porcine auto-transplanted kidneys. Apoptotic cell death by TUNEL positive cell staining (A, scale bars represents $30 \mu \mathrm{m}$ ) and inflammatory ED1 positive cells infiltrated in graft by immunofluorescence staining (B, scale bars represents $40 \mu \mathrm{m}$ ) in normal diet (a) or high-fat (b) groups, 3 months after renal auto-transplantation surgery. ${ }^{*} \mathrm{p}<0.05 \mathrm{vs}$. normal diet group; $\mathrm{n}=5$ in each group

TSP-1 is upregulated in the kidney from diet-induced obese mice with marked accumulation of TSP-1 in the glomeruli mesangium and tubular system [12]. They also demonstrated that TSP-1 deletion protects mice from obesity-induced renal fibrosis [12]. This detrimental effect was also observed in a syngeneic rat renal transplantation model. Briefly, a CD47 monoclonal antibody, which blocks the interaction between the ligand TSP-1 and its receptor $\mathrm{CD} 47$, used during the donor kidney perfusion, induced a marked improvement in post transplant survival [24]. These results were also observed in a renal ischemia reperfusion injury model [25]. In this study, we investigated the early effects of high-fat diet on pro-angiogenic pathway inhibition and TSP-1 expression in a porcine model of renal auto-transplantation with high clinical relevance. This allowed us to focus on $\mathrm{I} / \mathrm{R}$ injury in the absence of any allogeneic response and easily extrapolate the results to human as porcine and human kidneys have the same multilobular architecture and similar cortical microcirculation [26]. Hypercholesterolemia is associated with increased circulating levels of OxLDL [4]. These oxidized lipoproteins are involved in endothelial cell dysfunction [5], the first cell type subjected to I/R injury in organ transplantation. However, vascular endothelial cells are protected by the release of autocrine signaling molecules such as the pivotal VEGF$\mathrm{A}$ and are well known to proliferate, migrate and regenerate the injured tissue [9]. In a previous study, Sunitinib, a tyrosine kinase inhibitor which inhibits both VEGF and PDGF receptors, prevents chronic rejection changes in experimental kidney transplantation and preserved significantly renal graft function after transplantation highlighting the role of VEGF in early kidney graft function [27].

In the first part of this study, we investigated in endothelial cell model, the effects of OxLDL incubation on TSP-1 expression. We reported, for the first time, that OxLDL promotes TSP-1 secretion in endothelial artery cells according to the increase expression of ADAMTS- 1 and decreases the expression of alpha5beta3 integrin known as an integrin required for angiogenesis and endothelial proliferation [28]. TSP-1 uses multiple pathways to modulate endothelial cell migration [29]. TSP-1 is known as a natural antagonist of alpha5beta3 integrin through alpha5beta3 ligation and it has been shown to negatively regulate focal adhesion formation on fibronectin, suggesting that integrin function may be impaired [29]. These results suggest a repressive role of OxLDL production stimulated by hyperlipidic diet on angiogenesis through a TSP-1-dependent pathway underlining by the SiRNA experiment. Renal I/R induces regenerative processes such as angiogenesis which could be affected by OxLDL permanent production. Since our in vitro model relies on the culture on one cell type and is far to mimic the complexity of the kidney physiology, we decided to further test our hypothesis in our porcine 
Table 3 Patient characteristics

\begin{tabular}{|c|c|c|}
\hline & $\begin{array}{l}\text { Normocholesterolemia } \\
\text { Donors }\end{array}$ & $\begin{array}{l}\text { Hypercholesterolemia } \\
\text { Donors }\end{array}$ \\
\hline Age (years) & $43 \pm 9$ & $53 \pm 11$ \\
\hline Traumatic death $(\%, \mathrm{n})$ & $29(2)$ & $33(3)$ \\
\hline Hemorrhagic and ischemic stroke $(\%, n)$ & $71(5)$ & $67(6)$ \\
\hline Anastomosis time (min) & $42 \pm 12$ & $43 \pm 9$ \\
\hline Cold ischemia time (min) & $888 \pm 87$ & $870 \pm 180$ \\
\hline \multirow[t]{2}{*}{ eGFR } & $81 \pm 8$ & $81 \pm 6$ \\
\hline & Recipients & Recipients \\
\hline Number & 7 & 9 \\
\hline Age (years) & $45 \pm 15$ & $50 \pm 14$ \\
\hline Male gender $(\%, n)$ & $86(6)$ & $56(5)$ \\
\hline Dialysis duration (months) & $16 \pm 9$ & $6 \pm 13^{*}$ \\
\hline Current smoker $(\%, \mathrm{n})$ & $71(5)$ & $22(2)^{*}$ \\
\hline Diabetes mellitus $(\%, n)$ & $14(1)$ & $0(0)$ \\
\hline Hypertension $(\%, n)$ & $86(6)$ & $78(7)$ \\
\hline Statin $(\%, n)$ & $57(4)$ & $44(4)$ \\
\hline Cholesterolemia the day before surgery $(\mathrm{mmol} / \mathrm{L})$ & $3.80 \pm 0.54$ & $6.04 \pm 1.08^{*}$ \\
\hline Cholesterolemia at 3 months (mmol/L) & $4.80 \pm 0.47$ & $5.78 \pm 1.48$ \\
\hline Cholesterolemia at 6 months (mmol/L) & $4.32 \pm 1.13$ & $4.91 \pm 1.19$ \\
\hline Cholesterolemia at 12 months (mmol/L) & $4.72 \pm 1.09$ & $5.10 \pm 0.50$ \\
\hline eGFR at 1 month & $48 \pm 17$ & $45 \pm 25$ \\
\hline eGFR a at 3 months & $53 \pm 23$ & $50 \pm 18$ \\
\hline eGFR at 12 months & $64 \pm 13$ & $44 \pm 13^{*}$ \\
\hline Urinary ratio protein/creatinine $(\mathrm{mg} / \mathrm{mmol})$ at 3 month & $31.4 \pm 21.2$ & $73.9 \pm 42.5$ \\
\hline Urinary ratio protein/creatinine $(\mathrm{mg} / \mathrm{mmol})$ at 12 month & $16.7 \pm 5.2$ & $78.6 \pm 80.4^{*}$ \\
\hline
\end{tabular}

Values are mean $\pm S D$

${ }^{*} p<0.05$ vs. normocholesterolemia

a eGFR: estimated glomerular filtration rate according to the MDRD formula ( $\left.\mathrm{mL} / \mathrm{min} / 1.73 \mathrm{~m}^{2}\right)$

model of renal auto-transplantation modulated only by the effect of the diet.

In previous studies, it has been shown that high-fat diet affects renal VEGF-A protein expression and impairs microvascular structures in native porcine kidney without ischemic insults [30,31]. This aspect is well documented and suggests that high-fat diet in transplant condition could affect pro-angiogenic processes mediated by VEGF-A. We exclusively focused this study on the role of high-fat diet in early vascular regenerative processes after an $\mathrm{I} / \mathrm{R}$ injury in renal transplantation and excluded the effect of high-fat diet in native kidney since it was previously reported $[30,31]$. In pigs fed with a high-fat diet beginning from weaning up, we previously reported an increase in plasma levels of OxLDL at the day of surgery and at 3 months after transplantation [15]. This later increase was concomitant with a rise of total cholesterol levels. The discrepancy between plasma cholesterol and OxLDL levels the day of surgery could be explained by the fast required before surgery and underlined the role of high levels of plasma OxLDL during the early step of reperfusion. During follow up, renal function assessed by creatinine clearance was similar in both groups. This monitoring window for 3 months might be too short to observe significant differences in graft function. However, we observed a significant elevated proteinuria in high-fat diet group at 3 months post-transplantation which is described as a pivotal time point to investigate tissue and vascular remodeling and pro-angiogenic pathways $[10,17]$.

Renal tissue ischemia is one crucial factor in the development and progression of chronic kidney disease in general. It is known that HIFla expression is increased in area of severe fibrosis as observed in high-fat diet group indicated by Masson trichrome staining [9]. Decreased oxygen tension activates the stabilization of HIF1a, inducing a large range of pro-survival responses such as VEGF-A expression [32]. However, in this study, high-fat diet promotes a HIF1a stabilization without observed transcriptional effects on VEGF-A expression. 

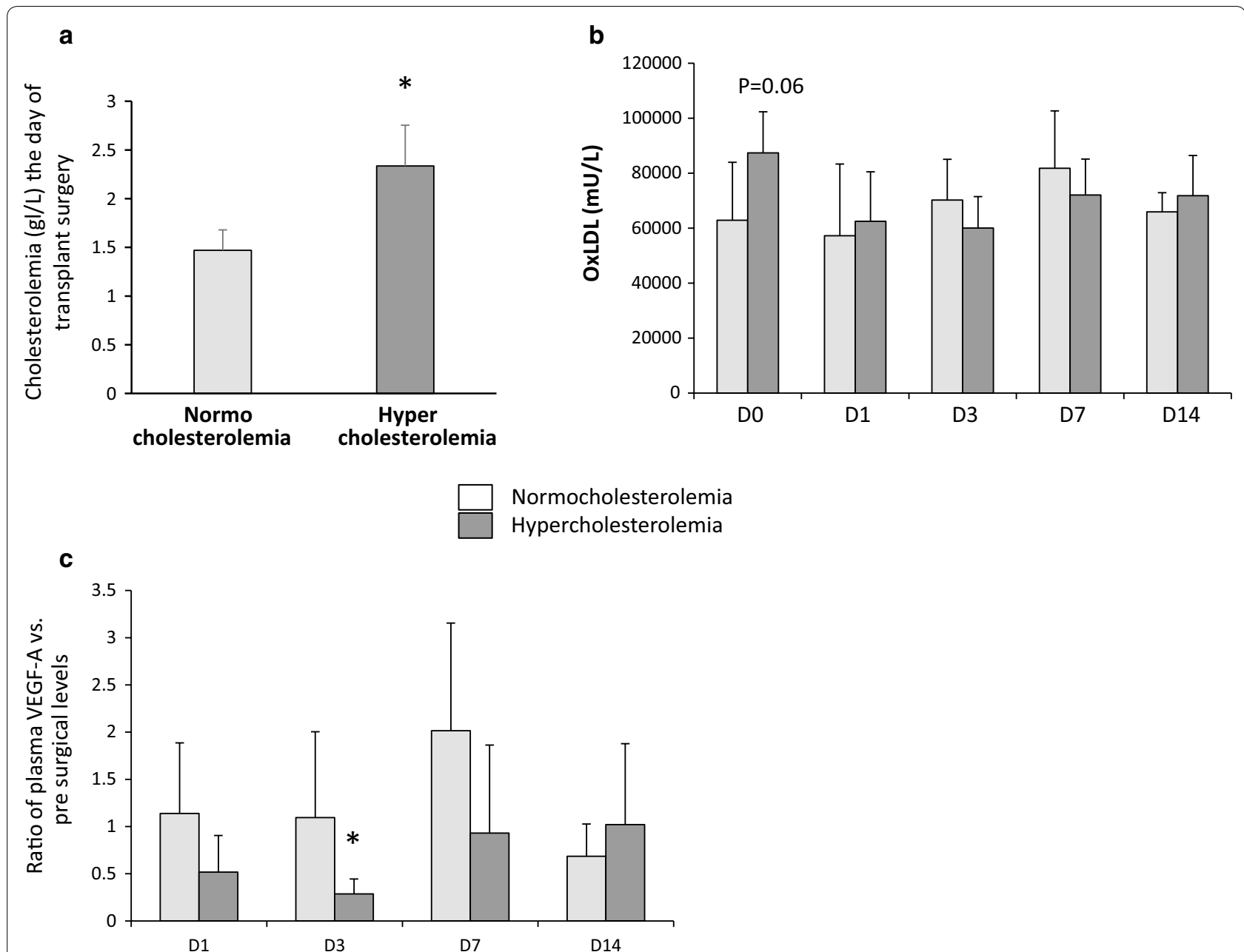

Fig. 8 Hypercholesterolemia in renal transplanted patient is associated with an early down regulation of plasmatic VEGF-A. Patients were classified in two groups in relation to their plasma cholesterol levels the day before renal transplantation $(\mathbf{a} ; n=7-9)$. OxLDL levels were measured in the short term follow up of patients ( 2 weeks after transplantation) in plasma $(\mathbf{b} ; n=3-9)$ as well as VEGF-A $(\mathbf{c} ; n=3-8)$. Values significantly different from the hypocholesterolemic group are represented by ${ }^{*} p<0.05$

In addition, high-fat diet reduced the pro-angiogenic capacity of kidney graft as shown by the repressed expressions of SDF-1 and VEGF-A proteins [9, 28, 33]. Taken together, these results suggest an anti-angiogenic effect of hyperlipidic diet that could be mediated by TSP1. Neovascularization induced by angiogenic factors is counterbalanced by the effects of angiogenic inhibitors, such as TSP-1, a glycoprotein expressed at very low levels in the healthy renal cortex and upregulated during some renal diseases [12]. Thakar et al. previously reported that TSP-1 null mice exhibited a significant protection against renal ischemia supporting the fact that TSP-1 acts as a regulator of ischemic damages in the kidney [34]. TSP-1 inhibits angiogenesis through its direct effects on endothelial cells and indirect effects on growth-factor mobilization and activation [9]. To investigate the role of TSP-1 in our conditions, we evaluated its expression in grafts. Hyperlipidic diet induced an over-expression of TSP-1 protein, 3 months after transplantation, in glomerulus, intensively marked in tubules and interstitium without mRNA expression change suggesting a post-transcriptional regulation of TSP-1. This hypothesis was supported by ADAMTS-1 overexpression. Indeed, ADAMTS-1 is a soluble matrix metalloprotease molecule that inhibits angiogenesis by mechanisms that may involve direct sequestration of VEGF or release activation of anti-angiogenic thrombospondin-derived peptides such as TSP-1 $[35,36]$. ADAMTS-1 was already described to be enhanced in rat proximal tubules after $\mathrm{I} / \mathrm{R}$ [37] and also in atherosclerosis with macrophage invading the tissue [38]. We observed an increase of ED1-positive macrophages infiltrating interstitium in high-fat diet group, in accordance with the elevated level expression of ADAMTS-1. The significant increase of 
inflammatory ED-1 positive macrophages in the interstitium of kidney grafts exposed to high OxLDL levels was linked with the profibrotic milieu associated with tubular atrophy. In addition, in this group, TSP-1 could stimulate apoptotic process as suggested by the TUNEL staining increase [39]. In order to investigate the consequences of these pro-inflammatory and regenerative pathways on vascular remodeling, we characterized the cortical microvascularization damages. High-fat diet increased the microvascular rarefaction induced by $I / R$ injury in our renal transplantation model particularly for small vascular segments with diameter inferior to $40 \mu \mathrm{m}$ according to the decrease of capillary density and the inhibition of pro-angiogenic pathways observed. In addition, the decrease of vascular segment diameter supported a vascular remodeling according to the high ratio of media-to-lumen and fibrosis development.

In order to test the relevance of our results in human transplantation, we investigated the effect of hypercholesterolemia on the systemic levels of the pivotal angiogenic vascular growth factor: VEGF-A in the short-term follow up after renal transplantation. We observed that recipients with high levels of cholesterol the day of surgical procedure, exhibited a decrease of plasmatic levels of VEGF-A during the first 3 days after transplantation suggesting a reduction of the regenerative processes in the graft. In addition, in this preliminary study, we underlined that the level of cholesterolemia the day of surgical procedure could affect graft outcome as indicated by the decrease of filtration function (eGFR) and by the increase of urinary protein excretion in hypercholesterolemic patient, 1 year after reperfusion. Although patient characteristics were similar in both groups, we should notice that other confounding factors could be involved in these differences such as immunosuppressive therapies. Further analyses will be necessary to support the correlation between the increase of TSP-1 levels observed in plasma recipient before surgery and its role on plasma VEGF-A levels early after transplantation.

\section{Conclusions}

Taken together, these results support that: (1) OxLDL are involved in abrogation of regenerative capacity of kidney grafts (2) TSP-1 is overexpressed in porcine kidney grafts exposed to high-fat diet and associated to a repression of pro angiogenic pathways, (3) high-fat diet induces microvascular rarefaction and vascular remodeling and (4) hypercholesterolemic patients have an early decrease of VEGF-A plasma levels after transplantation surgery.

In conclusion, our study underlines the impact of a short term exposition to high-fat diet on the early stage of regenerative processes after renal transplantation, distinct from the chronic injury of atherosclerosis induced by dyslipidemia. In addition, high-fat diet or dyslipidemia in recipient of kidney graft could affect organ outcome identifying TSP-1 as a therapeutic target of interest and emphasizing the need to better control either cholesterol or OxLDL plasma levels in recipient at the early stage of renal transplantation.

\section{Additional files}

Additional file 1: Table S1. Primer sequences for RT-qPCR analysis in porcine kidneys.

Additional file 2: Figure S1. Expression of TSP1 using western blotting in HAEC (first lane) and in HAEC subjected to Si TSP1 (last lane).

Additional file 3: Figure S2. Negative control for immunohistochemistry of HIF1a in renal porcine tissue.

Additional file 4: Figure S3. Negative control for immunohistochemistry of VEGF in renal porcine tissue.

Additional file 5: Figure S4. Negative control for immunohistochemistry of TSP1 in renal porcine tissue.

Additional file 6: Figure S5. VEGFA, HIF1a, ADAMTS1 and TSP1 mRNA expression by real time quantitative PCR in high-fat or normal diet groups 3 months after auto-transplantation $(n=5-6)$.

Additional file 7. Supplementary material for aminopeptidase staining in renal porcine tissue and for plasma TSP1 quantification in human.

Additional file 8: Figure S6. Quantification of aminopeptidase by immunostaining from kidneys 3 months after auto-transplantation subjected or not to a hyperlipidic diet.

Additional file 9: Figure S7. Hypercholesterolemia is associated with an upregulation of plasmatic TSP-1. Patients were classified in two groups in relation to their plasma cholesterol levels the day before renal transplantation and TSP-1 levels were measured $(n=6-7)$. Values significantly different from the normocholesterolemic group are represented by ${ }^{*} p<0.05$.

\section{Abbreviations}

ADAMTS-1: a disintegrin and metalloproteinase with thrombospondin motif-1 aSMA: alpha-smooth muscle actin; eGFR: estimated glomerular filtration rate; HAEC: human aortic endothelial cells; HIF1a: hypoxia-inducible factor 1a; I/R: ischemia reperfusion; LDL: Iow density lipoprotein; MMP-9: matrix metalloproteinase 9; OxLDL: oxidized LDL; SDF-1: stromal cell-derived factor-1; TGF $\beta$ : transforming growth factor $\beta$; TSP-1: thrombospondin-1; VEGF-A: vascular endothelial growth factor-A.

\section{Authors' contributions}

TK analyzed and interpreted the patient data, carried out protein studies and performed the statistical analysis; FF analyzed and interpreted the experimental data, was a major contributor in writing the manuscript and was involved in conception and design of this study; TK carried out protein studies; SM carried out microCT studies; GA performed animal surgery; AT analyzed and interpreted the patient data; MD carried out microCT studies; EB carried out protein studies; CS carried out cell experimentations and has been involved in revising the manuscript critically for important intellectual content; $\mathrm{TH}$ analysed and interpreted histochemical and immunohistochemical data, drafted the manuscript and was involved in conception and design of this study. All authors read and approved the final manuscript.

\section{Author details}

${ }^{1}$ INSERM U1082 IRTOMIT, 2 rue de la Milétrie, CS90577, 86000 Poitiers, France.

${ }^{2}$ Service d'Anesthésie-Réanimation, CHU de Poitiers, 86000 Poitiers, France.

${ }^{3}$ Faculté de Médecine et de Pharmacie, Université de Poitiers, 86000 Poitiers, 
France. ${ }^{4}$ Faculté de Médecine, EA 6309 “Maintenance Myélinique et Neuropathies Périphériques», Université de Limoges, 87000 Limoges, France. ${ }^{5}$ Laboratoire de Biochimie et Génétique Moléculaire, CHU de Limoges, 87000 Limoges, France. ${ }^{6}$ Service Medico-Chirurgical de Pediatrie, CHU de Poitiers, 86000 Poitiers, France. ${ }^{7}$ Service de Chirurgie Cardio-Thoracique, CHU de Poitiers, 86000 Poitiers, France. ${ }^{8}$ Service de Néphrologie et Transplantation, CHU de Poitiers, 86000 Poitiers, France. ${ }^{9}$ UGCT-Department of Physics and Astronomy, Faculty of Sciences, Ghent University, Proeftuinstraat 86, 9000 Ghent, Belgium. ${ }^{10}$ Service de Biochimie, CHU de Poitiers, Poitiers 86000 France. ${ }^{11}$ IBiSA Plate-Forme MOdélisation Préclinique-Innovations Chirurgicale et Technologique (MOPICT)', Domaine Expérimental du Magneraud, 17700 Surgères, France. ${ }^{12}$ FHU SUPORT'SUrvival oPtimization in ORgan Transplantation', 86000 Poitiers, France.

\section{Acknowledgements}

We are grateful to William Hebrard (Département de Génétique Animale, INRA, Surgères), Sandrine Joffrion, Virginie Ameteau and Maite Jacquard for their excellent technical support.

\section{Competing interests}

The authors declare that they have no competing interests.

\section{Availability of data and materials}

The datasets used and/or analysed during the current study are available from the corresponding author on reasonable request.

\section{Consent for publication}

Not applicable.

\section{Ethics approval and consent to participate}

Renal auto-transplantation model was performed 2 months after weaning as previously described in accordance with the institutional committee for the use and care of laboratory animals (CEEA Poitou-Charentes CE2012-4).

\section{Funding}

This study was supported by research grants from Société de Néphrologie, Fondation Transplantation, and Conseil Régional Poitou-Charentes.

\section{Publisher's Note}

Springer Nature remains neutral with regard to jurisdictional claims in published maps and institutional affiliations.

Received: 2 October 2018 Accepted: 31 December 2018

Published online: 14 January 2019

\section{References}

1. Chade AR, Mushin OP, Zhu X, Rodriguez-Porcel M, Grande JP, Textor SC, Lerman A, Lerman LO. Pathways of renal fibrosis and modulation of matrix turnover in experimental hypercholesterolemia. Hypertension. 2005;46:772-9.

2. Grundy SM, Stone NJ, Bailey AL, Beam C, Birtcher KK, Blumenthal RS, Braun LT, de Ferranti S, Faiella-Tommasino J, Forman DE, et al. 2018 AHA/ACC/AACVPR/AAPA/ABC/ACPM/ADA/AGS/APhA/ASPC/NLA/ PCNA guideline on the management of blood cholesterol: a report of the American College of Cardiology/American Heart Association Task Force on Clinical Practice Guidelines. J Am Coll Cardiol. 2018. https://doi. org/10.1016/j.jacc.2018.11.002

3. Wilson PWF, Polonsky TS, Miedema MD, Khera A, Kosinski AS, Kuvin JT. Systematic Review for the 2018 AHA/ACC/AACVPR/AAPA/ABC/ACPM/ ADA/AGS/APhA/ASPC/NLA/PCNA guideline on the management of blood cholesterol: a report of the American College of Cardiology/American Heart Association Task Force on Clinical Practice Guidelines. J Am Coll Cardiol. 2018. https://doi.org/10.1016/j.jacc.2018.11.004

4. Duarte MM, Rocha JB, Moresco RN, Duarte T, Da Cruz IB, Loro VL, Schetinger MR. Association between ischemia-modified albumin, lipids and inflammation biomarkers in patients with hypercholesterolemia. Clin Biochem. 2009;42:666-71.
5. Raman KG, Gandley RE, Rohland J, Zenati MS, Tzeng E. Early hypercholesterolemia contributes to vasomotor dysfunction and injury associated atherogenesis that can be inhibited by nitric oxide. J Vasc Surg. 2011;53:754-63.

6. Basile DP. Rarefaction of peritubular capillaries following ischemic acute renal failure: a potential factor predisposing to progressive nephropathy. Curr Opin Nephrol Hypertens. 2004;13:1-7.

7. Basile DP. The endothelial cell in ischemic acute kidney injury: implications for acute and chronic function. Kidney Int. 2007;72:151-6.

8. Cicco G, Panzera PC, Catalano G, Memeo V. Microcirculation and reperfusion injury in organ transplantation. Adv Exp Med Biol. 2005;566:363-73.

9. Tanaka T, Nangaku M. Angiogenesis and hypoxia in the kidney. Nat Rev Nephrol. 2013;9:211-22.

10. Rossard L, Favreau F, Giraud S, Thuillier R, Le Pape S, Goujon JM, Valagier $A$, Hauet T. Role of warm ischemia on innate and adaptive responses in a preclinical renal auto-transplanted porcine model. J Transl Med. 2013;11:129.

11. Rossard L, Favreau F, Demars J, Robert R, Nadeau C, Cau J, Thuillier R, Hauet T. Evaluation of early regenerative processes in a preclinical pig model of acute kidney injury. Curr Mol Med. 2012;12:502-5.

12. Cui W, Maimaitiyiming H, Qi X, Norman H, Wang S. Thrombospondin 1 mediates renal dysfunction in a mouse model of high-fat diet-induced obesity. Am J Physiol Renal Physiol. 2013;305:F871-80.

13. Hugo C, Daniel C. Thrombospondin in renal disease. Nephron Exp Nephrol. 2009;111:e61-6.

14. Adams JC, Lawler J. The thrombospondins. Int J Biochem Cell Biol. 2004;36:961-8.

15. Chatauret N, Favreau F, Giraud S, Thierry A, Rossard L, Le Pape S, Lerman LO, Hauet T. Diet-induced increase in plasma oxidized LDL promotes early fibrosis in a renal porcine auto-transplantation model. J Transl Med. 2014;12:76.

16. Favreau F, Thuillier R, Cau J, Milin S, Manguy E, Mauco G, Zhu X, Lerman LO, Hauet T. Anti-thrombin therapy during warm ischemia and cold preservation prevents chronic kidney graft fibrosis in a DCD model. Am J Transplant. 2007;10:30-9.

17. Favreau F, Zhu XY, Krier JD, Lin J, Warner L, Textor SC, Lerman LO. Revascularization of swine renal artery stenosis improves renal function but not the changes in vascular structure. Kidney Int. 2010;78:1110-8.

18. Hauet T, Goujon JM, Baumert H, Petit I, Carretier M, Eugene M, Vandewalle A. Polyethylene glycol reduces the inflammatory injury due to cold ischemia/reperfusion in autotransplanted pig kidneys. Kidney Int. 2002;62:654-67.

19. Jayle C, Favreau F, Zhang K, Doucet C, Goujon JM, Hebrard W, Carretier M, Eugene M, Mauco G, Tillement JP, Hauet T. Comparison of protective effects of trimetazidine against experimental warm ischemia of different durations: early and long-term effects in a pig kidney model. Am J Physiol Renal Physiol. 2007;292:F1082-93.

20. Zhu XY, Chade AR, Rodriguez-Porcel M, Bentley MD, Ritman EL, Lerman A, Lerman LO. Cortical microvascular remodeling in the stenotic kidney: role of increased oxidative stress. Arterioscler Thromb Vasc Biol. 2004;24:1854-9.

21. Feldkamp L, Davis L, Kress J. Practical cone-beam algorithm. JOSA A. 1984;1:612-9.

22. Maiga S, Allain G, Hauet T, Roumy J, Baulier E, Scepi M, Dierick M, Van Hoorebeke L, Hannaert P, Guy F, Favreau F. Renal auto-transplantation promotes cortical microvascular network remodeling in a preclinical porcine model. PLOS ONE. 2017;12:e0181067.

23. European best practice guidelines for renal transplantation. Section IV: Long-term management of the transplant recipient. IV.13 Analysis of patient and graft survival. Nephrol Dial Transplant. 2002;17(4):60-7.

24. Lin Y, Manning PT, Jia J, Gaut JP, Xiao Z, Capoccia BJ, Chen CC, Hiebsch RR, Upadhya G, Mohanakumar T, et al. CD47 blockade reduces ischemia-reperfusion injury and improves outcomes in a rat kidney transplant model. Transplantation. 2014;98:394-401.

25. Yao M, Rogers NM, Csanyi G, Rodriguez Al, Ross MA, St Croix C, Knupp $\mathrm{H}$, Novelli EM, Thomson AW, Pagano PJ, Isenberg JS. Thrombospondin-1 activation of signal-regulatory protein-alpha stimulates reactive oxygen species production and promotes renal ischemia reperfusion injury. J Am Soc Nephrol. 2014;25:1171-86. 
26. Giraud S, Favreau F, Chatauret N, Thuillier R, Maiga S, Hauet T. Contribution of large pig for renal ischemia-reperfusion and transplantation studies: the preclinical model. J Biomed Biotechnol. 2011;2011:532127.

27. Rintala JM, Savikko J, Palin N, Rintala SE, Koskinen PK, von Willebrand E. Oral platelet-derived growth factor and vascular endothelial growth factor inhibitor sunitinib prevents chronic allograft injury in experimental kidney transplantation model. Transplantation. 2016;100:103-10.

28. Brooks PC, Clark RA, Cheresh DA. Requirement of vascular integrin alpha v beta 3 for angiogenesis. Science. 1994;264:569-71.

29. Ashton AW, Cheng Y, Helisch A, Ware J. Thromboxane A2 receptor agonists antagonize the proangiogenic effects of fibroblast growth factor-2: role of receptor internalization, thrombospondin-1, and alpha(v)beta3. Circ Res. 2004;94:735-42.

30. Chade AR, Lerman A, Lerman LO. Kidney in early atherosclerosis. Hypertension. 2005:45:1042-9.

31. Chade AR, Bentley MD, Zhu X, Rodriguez-Porcel M, Niemeyer S, AmoresArriaga B, Napoli C, Ritman EL, Lerman A, Lerman LO. Antioxidant intervention prevents renal neovascularization in hypercholesterolemic pigs. J Am Soc Nephrol. 2004;15:1816-25.

32. Schley G, Klanke B, Schodel J, Forstreuter F, Shukla D, Kurtz A, Amann K, Wiesener MS, Rosen S, Eckardt KU, et al. Hypoxia-inducible transcription factors stabilization in the thick ascending limb protects against ischemic acute kidney injury. J Am Soc Nephrol. 2011;22:2004-15.
33. Liekens S, Schols D, Hatse S. CXCL12-CXCR4 axis in angiogenesis, metastasis and stem cell mobilization. Curr Pharm Des. 2010;16:3903-20.

34. Thakar CV, Zahedi K, Revelo MP, Wang Z, Burnham CE, Barone S, Bevans S, Lentsch AB, Rabb H, Soleimani M. Identification of thrombospondin 1 (TSP-1) as a novel mediator of cell injury in kidney ischemia. J Clin Invest. 2005;115:3451-9.

35. Lee NV, Sato M, Annis DS, Loo JA, Wu L, Mosher DF, Iruela-Arispe ML. ADAMTS1 mediates the release of antiangiogenic polypeptides from TSP1 and 2. EMBO J. 2006:25:5270-83.

36. Luque A, Carpizo DR, Iruela-Arispe ML. ADAMTS1/METH1 inhibits endothelial cell proliferation by direct binding and sequestration of VEGF165. J Biol Chem. 2003;278:23656-65.

37. Basile DP, Fredrich K, Chelladurai B, Leonard EC, Parrish AR. Renal ischemia reperfusion inhibits VEGF expression and induces ADAMTS-1, a novel VEGF inhibitor. Am J Physiol Renal Physiol. 2008;294:F928-36.

38. Ashlin TG, Kwan AP, Ramji DP. Regulation of ADAMTS-1, -4 and -5 expression in human macrophages: differential regulation by key cytokines implicated in atherosclerosis and novel synergism between TL1A and IL-17. Cytokine. 2013;64:234-42.

39. Guo N, Krutzsch HC, Inman JK, Roberts DD. Thrombospondin 1 and type I repeat peptides of thrombospondin 1 specifically induce apoptosis of endothelial cells. Cancer Res. 1997;57:1735-42.
Ready to submit your research? Choose BMC and benefit from:

- fast, convenient online submission

- thorough peer review by experienced researchers in your field

- rapid publication on acceptance

- support for research data, including large and complex data types

- gold Open Access which fosters wider collaboration and increased citations

- maximum visibility for your research: over 100M website views per year

At BMC, research is always in progress.

Learn more biomedcentral.com/submissions 